\title{
Patient satisfaction and gender composition of physicians - a cross-sectional study of community health services in Hubei, China
}

\author{
Change Xiong $^{1 *}$, Xiao Chen ${ }^{2 *}$, Xinyuan Zhao ${ }^{3}$ and Chaojie Liu ${ }^{4}$
}

\begin{abstract}
Background: Patient satisfaction is associated with both individual (patients and health workers) and organizational (health facilities) characteristics. This study aimed to establish a link between patient satisfaction and gender composition of physicians in community health service (CHS) organizations.

Methods: Participants were selected through multistage stratified random sampling: 36 CHS centers were selected from six municipalities in Hubei, China. All physicians on duty and patients visiting the CHS during the study period (from April to October in 2015) were invited to participate in this study: 324 physicians and 865 patients completed a questionnaire survey. Multilevel linear regression analyses were performed to determine the associations of patient satisfaction (scored from 1 to 5) with patient characteristics (gender, age, education, income, medical expense, frequency of visits to the CHS) and organizational features of the CHS (sex ratio of physicians, and gender differences of physicians in education and job satisfaction).

Results: Older patients and those with a higher medical bill had a lower degree of satisfaction $(p<0.05)$. At the organizational level: a higher proportion of male physicians weakened the negative association between patient age and patient satisfaction ( $p<0.05)$; a larger gap in university qualifications between male and female physicians exacerbated the negative association between patient age and patient satisfaction $(p<0.05)$.

Conclusions: The gender composition of physicians in CHSs is associated with patient satisfaction in the Chinese context: a larger gap (in number and qualifications) between male and female physicians is associated with higher patient satisfaction. Further studies are needed to explore the cultural roots of such an association.
\end{abstract}

Keywords: Patient satisfaction, Community health service, Gender composition

\section{Background}

Patient satisfaction has been commonly used for evaluating health care services. It reflects the overall experience of a patient [1]. Patient satisfaction affects clinical outcomes, patient retention, and patient-doctor relationships [2]. Satisfied patients are more likely to comply with medical advice and be cooperative. In China, great

\footnotetext{
*Correspondence: xche77@163.com; 11029360@qq.com

${ }^{1}$ Change Xiong, Department of Social Medicine and Health Management, School of Basic Medical Science, Hubei University of Science and

Technology, 88 Xianning Road, Xianning 437100, People's Republic of China

${ }^{2}$ Xiao Chen, Institute of Medicine, Hubei University of Science and

Technology, 88 Xianning Road, Xianning 437100, People's Republic of China

Full list of author information is available at the end of the article
}

efforts have been made to improve patient satisfaction with primary care [3], with an intention to divert patients from hospitals (often a very crowded environment) to community health services (CHSs) [4].

Patient satisfaction is shaped by at least four factors: the personal preference of the patient, the patient's expectation, the response tendency of the patient due to personal characteristics, and the quality of care received [1]. Some researchers argue that patient satisfaction can also be influenced by the previous experience of the patient and the opinions of others, such as relatives and friends [5]. 
Extensive studies have been undertaken to identify the determinants of patient satisfaction [6-23]. A study in the USA found that older and literacy-deficient patients tend to have a higher degree of satisfaction than their younger and functionally literate peers [10]. Cohen in the UK also found that age is a strong predictor of patient ratings on satisfaction for both hospital and primary care, with older patients having a higher degree of satisfaction $[8,13]$. This could be caused by the lower expectations of older patients $[14,18]$. But some researchers argue that older patients are also likely to be treated with more respect by physicians [15]. The findings on gender differences in patient satisfaction are mixed, with some claiming that female patients have a higher degree of satisfaction and others reaching an opposite conclusion $[13,14]$.

Patient satisfaction can also be shaped by the characteristics and behaviors of health workers [8-10, 16]. Good communication and attitudes (such as understanding, listening, respect and empathy) can increase the degree of patient satisfaction $[6,17]$. Compared with female physicians, male physicians tend to be more active and controlling in communications [18]. In some cultures, patients talk more, disclose more private information and make more positive statements to female physicians than to male physicians [19]. Harbinder [20] found that the gender of medical practitioners is associated with length of consultation, agendas elicited, and the content and style of communications. Cypress [21] reported that female doctors spend more time with female patients than with male patients. Studies in Western countries revealed that a female-sex role congruent communication style leads to a higher degree of patient satisfaction when women see a female physician [22, 23].

Patient satisfaction has been increasingly used in comparing the performance of healthcare organizations $[24,25]$. Indeed, patient satisfaction is a reflection of the collective efforts made by a healthcare organization. Each patient is looked after by a team comprising physicians, nurses and other professional and administrative workers. Strong teamwork can improve the patient experience [26-28]. The strength of an organizational culture which puts patients at the center of care is positively associated with patient satisfaction [29]. Young and colleagues [30] found that larger and urban hospitals are usually rated lower by patients in satisfaction surveys compared with smaller and rural hospitals. Services involving graduate medical students may also lower patient satisfaction [31].

It is not clear whether the gender composition of the medical workforce, an important organizational feature, may influence patient satisfaction. Given the fact that gender differences exist among individual medical workers in responding to patient needs [32, 33], it is reasonable to expect an association between patient satisfaction and the gender composition of medical workers in an organization. However, it is important to note that the role of gender in different cultures may vary considerably.

This study was undertaken in China, with an aim to establish associations between patient satisfaction and the gender composition of physicians in $\mathrm{CHS}$ organizations.

We hypothesized that patient satisfaction is a multiattribute construct, reflecting the experience of a patient over the process of interacting with a range of health workers and services in an organization. It is associated with the individual characteristics of the patient and the collective characteristics of the organization.

- Hypothesis 1: Patient satisfaction varies with the individual characteristics of patients, such as age, gender, education, and financial affordability of health care (measured by medical expenses and average household income);

- Hypothesis 2: Organizational characteristics as indicated by gender composition (in numbers, qualifications, and job satisfaction) in an organization moderates the effects of patient individual characteristics on patient satisfaction.

We did not assume a particular direction in the associations between patient satisfaction and organizational and patients' individual characteristics because such associations often depend on the system environment in which services are delivered.

\section{Methods \\ Setting}

From 2009 to 2012, the number of male registered physicians employed by $\mathrm{CHSs}$ in China increased from $41,250(46.8 \%)$ to $63,133(48.1 \%)$, while female registered doctors increased from 46,947 (53.2\%) to 78,750 (51.9\%) [34]. Compared with developed nations such as Australia [35], Canada [36], the UK [37] and the USA [38], China has a higher proportion of female physicians working in primary care settings. Moreover, not all physicians in China hold a university medical degree. About $37 \%$ of physicians in CHSs completed a bachelor degree of medicine.

This study was conducted in Hubei province. Hubei is situated in central China, with a population of 58.5 million. It ranks in the middle range of economic development in all the provinces in China in terms of per capita GDP. There are 771 hospitals and 326 CHS centers, which employ about 65,800 and 5600 physicians, respectively [39]. 


\section{Sampling}

A multistage stratified random sampling strategy was employed in this study. Seventeen municipalities in Hubei were divided into three economic zones according to per capita GDP. Two municipalities from each zone were randomly selected. Six CHS organizations were then randomly selected from each participating municipality. This resulted in a total sample of $36 \mathrm{CHS}$ centers.

\section{Data collection}

The number of male and female physicians was provided by the managers of the participating CHSs.

All physicians on duty and the patients visiting the participating $\mathrm{CHS}$ on the day when the survey was undertaken during the period from April to October of 2015 were invited to participate in a questionnaire survey. A total of 1319 questionnaires were dispatched: 387 to physicians and 932 to patients. Response rates were high, with 324 (83.7\%) questionnaires being returned from the invited physicians and 865 (92.8\%) questionnaires being returned from the invited patients. The questionnaire was administered through face-to-face interviews by six trained interviewers. Patients were interviewed after they had completed a visit to the CHS.

The questionnaire for physicians consisted of two parts. Part one collected information related to sociodemographic characteristics (e.g. age and sex) and professional training (e.g. qualification). Part two assessed job satisfaction. Previous studies found that the job satisfaction of medical workers is positively associated with patient satisfaction [40, 41]. The job satisfaction scale used in this study was adapted from the Minnesota Satisfaction Questionnaire (MSQ), which contains 12 items [42]. The MSQ has been validated and widely accepted internationally [43]. Example questions included how the respondents felt about the "decision-making ability of their superior" and their "job promotion opportunities". Respondents were asked to rate their experiences on a five-point Likert scale [from very dissatisfied (score 1 ) to very satisfied (score 5)]. The item scores were added and averaged. The internal consistency of the MSQ scale in this study population is high, with an alpha coefficient of 0.839 .

The questionnaire for patients also consisted of two parts. Part one collected information related to the socio-demographic characteristics of patients, such as age, gender, education, household income and frequency of visits to CHSs in the past year. Part two assessed patient satisfaction towards "quality of care", "skills of physicians" and "attitudes of physicians". Each of the three aspects was measured using one item: "Overall, how satisfied are you with the quality of care you received?" (quality of care); "How satisfied are you with the skills of your physicians who made the diagnoses and delivered treatment?" (skills of physicians); "How satisfied are you with the attitudes of your physicians?" (attitudes of physicians). Respondents (including 44 (5\%) respondents on behalf of their $<18$ years old children) were asked to rate items on a five-point Likert scale, ranging from 1 (strongly dissatisfied) to 5 (strongly satisfied). These items were chosen from the Patient Satisfaction Questionnaire Short-form (PSQ-18) [44] with consideration of their relevance and importance to $\mathrm{CHS}$ patients in China. Sixma and colleagues argue that patient expectations and concerns should be the utmost consideration in measuring patient satisfaction [45]. In general, patient expectations encompass a variety of dimensions, which range from the physical facility and convenience to skilled services and outcomes of care [3, 46, 47]. In response to patient expectations, health care services need to be delivered in a fair, compassionate and respectful way. Hall and Dornan [48] found that services that demonstrate humanness and competence, cost-effectiveness, good accessibility and share information are most valued by patients. Studies in China showed that the overwhelming concerns of CHS patients rest on quality of care, skill competency of physicians, and attitudes of health workers $[49,50]$.

\section{Outcome variable}

Patient satisfaction was measured by the average score of the three items, which had an acceptable internal consistency (alpha $=0.715)$.

\section{Independent variable}

The gender composition of the medical workforce in CHSs is the major focus of this study. Three variables were generated to measure gender composition. The sex ratio (number of males to females) reflects the percentage distribution of gender in relation to physicians. We also measured differences in qualifications and job satisfaction between male and female physicians. We used the questionnaire for physicians to estimate the sex ratio in relation to the percentage of physicians with a university qualification. Gender differences in job satisfaction were measured by the difference in mean scores between male and female physicians.

A number of factors may have a confounding influence on the associations between patient satisfaction and the gender composition of physicians. In this study, we considered patient age, gender, education, average household monthly income, medical expenses relating to recent visits to a CHS, and the frequency of visits to a $\mathrm{CHS}$ over the past year.

\section{Data analysis}

Multi-level linear regression analyses were performed using HLM7.0. We established a two-level random effect 
models using maximum likelihood estimation (MLE). The two levels of measurements corresponded to the individual characteristics of patients and the collective characteristics of CHSs, respectively (Table 1).

We started with an unconditional model (intercept only, Model One in Table 3) to examine the variations in the outcome variable (patient satisfaction) first.

We then developed a model (Model Two) to test hypothesis one, containing only level one variables (patient characteristics):

$$
\begin{aligned}
\mathrm{Y}_{\mathrm{ij}}= & \beta_{0 \mathrm{j}}+\beta_{1 \mathrm{j}}(\text { gender })+\beta_{2 \mathrm{j}}(\text { age })+\beta_{3 \mathrm{j}}(\text { education }) \\
& \left.+\beta_{4 \mathrm{j}}(\text { income })+\beta_{5 \mathrm{j}} \text { (medical expense }\right) \\
& +\beta_{6 \mathrm{j}}(\text { frequency of visits to } \mathrm{CHS})+\gamma_{\mathrm{ij}}
\end{aligned}
$$

Level two variables (organizational characteristics) were subsequently introduced into the model to estimate the mixed effect regression coefficients. Because patient gender, education, household income and frequency of visits to CHS did not appear as a significant predictor of patient satisfaction, their mixed effects with level two variables were not explored. Model Three tested the interaction effect between "age" and level two variables, while Model Four tested the interaction effect between "medical expense" and level two variables (hypothesis two).

$$
\begin{aligned}
& \beta_{0 j}=\gamma_{00}+\mu_{0 j} \\
& \beta_{1 j}=\gamma_{10}+\mu_{1 j}
\end{aligned}
$$

\begin{tabular}{|c|c|}
\hline Variable & Description and value \\
\hline \multicolumn{2}{|l|}{ Individual-level (patients) } \\
\hline Sex & $0=$ Male $;=$ Female \\
\hline Age & Continuous variable measured as "years" \\
\hline Education & $\begin{array}{l}0=\text { Without a tertiary qualification; } 1=\text { With a } \\
\text { tertiary qualification }\end{array}$ \\
\hline $\begin{array}{l}\text { Average monthly } \\
\text { household income }\end{array}$ & $\begin{array}{l}\text { Continuous variable measured in monetary } \\
\text { terms "Yuan" }\end{array}$ \\
\hline Medical expenses & $\begin{array}{l}\text { Continuous variable measured in monetary } \\
\text { terms "Yuan" }\end{array}$ \\
\hline Frequency of visits & Continuous variable measured as "Times" \\
\hline \multicolumn{2}{|c|}{ Organizational-level (community health services) } \\
\hline Sex ratio "numbers" & $\begin{array}{l}\text { Ratio of the number of male to female } \\
\text { medical workers }\end{array}$ \\
\hline $\begin{array}{l}\text { Sex ratio } \\
\text { "qualifications" }\end{array}$ & $\begin{array}{l}\text { Percentage of male to female physicians with } \\
\text { a bachelor degree }\end{array}$ \\
\hline $\begin{array}{l}\text { Gender difference in } \\
\text { job satisfaction }\end{array}$ & $\begin{array}{l}\text { Difference in mean scores of job satisfaction } \\
\text { between male and female physicians }\end{array}$ \\
\hline
\end{tabular}

Table 1 Values assigned to the independent variables in multilevel modelling

$$
\begin{aligned}
\beta_{2 \mathrm{j}}= & \gamma_{20}+\gamma_{21}(\text { sex ratio" numbers") } \\
& +\gamma_{22}(\text { sex ratio qualifications") } \\
& +\gamma_{23}(\text { gender differences in job satisfaction }) \\
& +\mu_{2 \mathrm{j}} \\
\beta_{3 \mathrm{j}}= & \gamma_{30}+\mu_{3 \mathrm{j}} \\
\beta_{4 \mathrm{j}}= & \gamma_{40}+\mu_{4 \mathrm{j}} \\
\beta_{5 \mathrm{j}}= & \gamma_{50}+\gamma_{51}(\text { sex ratio “numbers" }) \\
& +\gamma_{52}(\text { sex ratio "qualifications" }) \\
& +\gamma_{53}(\text { gender differences in job satisfaction }) \\
& +\mu_{5 \mathrm{j}} \\
\beta_{6 \mathrm{j}}= & \gamma_{60}+\mu_{6 \mathrm{j}}
\end{aligned}
$$

$Y_{\mathrm{ij}}$ represents the satisfaction rating of patient $\mathrm{i}$ (level 1) in CHS $\mathrm{j}$ (level 2). $\beta$ is a fixed parameter to be estimated.yis a CHS-specific random effect. Mis the component of error term.

\section{Results}

\section{Characteristics of participants}

Patient respondents $(n=865)$ had an average age of 44.65 $(\mathrm{SD}=19.54)$ years: about $61 \%$ were female; and $22 \%$ had a university degree. Their average monthly household income was 3785 (SD 3466) Yuan RMB. On average, each patient visited a CHS five times in the past year and the most recent visit costed 464 Yuan RMB (Table 2).

Physician respondents $(n=324)$ had an average age of 38.24 ( $\mathrm{SD}=10.92)$ years: about $54 \%$ were female; and $42 \%$ held a middle or senior professional title. On average, they had 16.25 (SD = 12.50) years of work experience.

The 36 participating CHSs had an average sex ratio of 0.65 . Male physicians were more likely to hold a bachelor degree than their female colleagues (male to female ratio: 1.50). Male physicians had a significantly higher degree of job satisfaction than their female colleagues $(t=4.44, p=0.000)$.

\section{Patient satisfaction and multilevel modelling}

On average, patients gave a satisfaction score of 1.97 $(\mathrm{SD}=0.65)$ to CHSs out of a maximum possible score of five. The unconditional model (Model One) revealed statistically significant variability in the outcome variable (patient satisfaction) across CHSs $(p<0.001$, Table 3$)$. The subsequent multilevel models indicated that heterogeneity in patient satisfaction scores existed across CHS organizations as measured by intraclass correlation coefficients (ICC). The ICC values range from 0 to 1 . A nonzero ICC value is an indication of heterogeneity. The ICC values showed that organizational (level two) effects contributed to about $5.77 \%$ of the total variance in patient satisfaction. A reliability of 0.543 for Model One 
Table 2 Characteristics of patient respondents and participating community health services (CHS)

\begin{tabular}{|c|c|c|c|c|c|}
\hline Variable & Percentage & Mean & S.D. & Min. & Max. \\
\hline \multicolumn{6}{|l|}{ Individual-level (patients) } \\
\hline Proportion of female respondents & $61 \%$ & & & & \\
\hline Age (Years) & & 44.65 & 19.54 & 1 & 96 \\
\hline Percentage of respondents with a university degree & $22 \%$ & & & & \\
\hline Average monthly household income (Yuan) & & 3785 & 3466 & 100 & 50,000 \\
\hline Medical expense (Yuan) & & 464 & 898 & 2 & 8700 \\
\hline Frequency of visits to CHS & & 5.07 & 8.11 & 1 & 99 \\
\hline \multicolumn{6}{|l|}{ Organizational-level (community health services) } \\
\hline Sex ratio (Male to Female) - numbers of physicians & & 0.65 & 1.14 & 0 & 7.00 \\
\hline Sex ratio (Male to Female) - percentage of physicians with a university degree & & 1.50 & 1.16 & 0 & 6.00 \\
\hline Gender difference in job satisfaction scores & & 0.7 & 4.83 & -7.67 & 12.40 \\
\hline
\end{tabular}

means that each sample of CHS is a reliable estimate of the population mean. Thus, a continued multi-level analysis is appropriate.

In the regression model containing level one variables only (Model Two in Table 3), patient age and medical expenses relating to a recent visit to a CHS appeared to be significant predictors of patient satisfaction. Older patients and those with a higher medical bill had a lower degree of satisfaction $(p<0.05)$.
When level two variables were incorporated into the regression model for their interaction effects with level one variables "age" (Model Three) and "medical expense" (Model Four), the effect sizes of level one variables changed significantly (Table 3 ). The two-level mixed effect models showed that the negative association between age and patient satisfaction varied with the gender composition of physicians in CHSs; but the negative association between medical expenses and

Table 3 Determinants of patient satisfaction scores - multilevel linear regression analyses

\begin{tabular}{|c|c|c|c|c|c|c|c|c|}
\hline \multirow[t]{2}{*}{ Independent variables } & \multicolumn{2}{|c|}{ Model One } & \multicolumn{2}{|c|}{ Model Two } & \multicolumn{2}{|c|}{ Model Three } & \multicolumn{2}{|c|}{ Model Four } \\
\hline & Coef. & SE & Coef. & SE & Coef. & SE & Coef. & SE \\
\hline \multicolumn{9}{|l|}{ Fixed effects } \\
\hline Intercept $\left(\beta_{0}\right)$ & & & 1.970 & 0.035 & 1.970 & 0.035 & 1.970 & 0.034 \\
\hline \multicolumn{9}{|l|}{ Individual-level (patients) } \\
\hline $\operatorname{Sex}\left(\beta_{1}\right)$ & & & -0.031 & 0.041 & -0.024 & 0.040 & -0.031 & 0.041 \\
\hline Age $\left(\beta_{2}\right)$ & & & $-0.005^{* *}$ & 0.001 & $-\overline{0.009^{* *}}$ & -0.002 & $-\overline{0.005^{* *}}$ & 0.001 \\
\hline Education $\left(\beta_{3}\right)$ & & & -0.025 & 0.061 & -0.033 & 0.060 & -0.029 & 0.061 \\
\hline Monthly household income $\left(\beta_{4}\right)$ & & & 0.000 & 0.000 & 0.000 & 0.000 & 0.000 & 0.000 \\
\hline Medical expenses for recent visit to $\mathrm{CHS}\left(\beta_{5}\right)$ & & & $-0.000^{*}$ & 0.000 & -0.000 & 0.000 & -0.000 & 0.000 \\
\hline Frequency of visits to $\mathrm{CHS}\left(\beta_{6}\right)$ & & & -0.000 & 0.004 & -0.000 & 0.004 & -0.001 & 0.004 \\
\hline \multicolumn{9}{|l|}{ Organizational-level (community health services) } \\
\hline Sex ratio (Male to Female) - numbers of physicians $\left(\beta_{i 1}\right)$ & & & & & $0.012^{*}$ & 0.005 & 0.001 & 0.000 \\
\hline $\begin{array}{l}\text { Sex ratio (Male to Female) - percentage of physicians with a university } \\
\text { degree }\left(\beta_{i 2}\right)\end{array}$ & & & & & $-0.001^{*}$ & 0.000 & -0.000 & 0.000 \\
\hline Gender difference in job satisfaction scores $\left(\beta_{\mathrm{i} 3}\right)$ & & & & & 0.000 & 0.000 & -0.000 & 0.000 \\
\hline \multicolumn{9}{|l|}{ Random effects } \\
\hline Variance component & & $\begin{array}{l}.0243 \\
\left(\mu_{0}\right)\end{array}$ & & .0235 & & $\begin{array}{l}.0237 \\
\left(\mu_{2}\right)\end{array}$ & & $\begin{array}{l}.0236 \\
\left(\mu_{5}\right)\end{array}$ \\
\hline$x^{2}(d f)$ & & 97.688 & & 90.604 & & 91.800 & & 91.212 \\
\hline$p$ & & $<0.001$ & & $\begin{array}{l}< \\
0.001\end{array}$ & & $<0.001$ & & $<0.001$ \\
\hline
\end{tabular}


patient satisfaction did not vary with the gender composition of physicians in CHSs.

The organizational difference in the gender composition of physicians contributed to $5.89 \%$ of the total variance in patient satisfaction scores (which was estimated using intercept variance component of 0.0237 divided by intercept variance component plus level one variance component $0.379+0.0237$ ). A higher percentage of male physicians weakened the negative effect of patient age on patient satisfaction. By contrast, a higher level of gender difference in qualifications exacerbated the negative effect of patient age on patient satisfaction.

\section{Discussion}

\section{Patient satisfaction is associated with patient age and medical expenditure}

This study revealed an overall low level of patient satisfaction with CHSs. In China, both hospitals and CHSs provide non-referred primary care to patients. It is not uncommon for patients to give lower ratings on CHSs in comparison with hospitals [11]. In addition, we found that older patients have a lower degree of satisfaction with CHSs than younger patients, which is different from the findings of some other studies [8, 9, 12-15]. Mitchell [9] argued that older patients are more enthusiastic about interacting with their physicians and therefore are more likely to be satisfied because their physicians are more likely to adopt a patient-centered approach with them. It is too early to jump to a conclusion about the reasons behind the lower degree of satisfaction of older patients in China. However, empirical studies show that patients of different ages prioritize different service dimensions in their satisfaction ratings. Older patients tend to value continuity more than younger patients do. In China, a shortage in the medical workforce is serious, which leads to heavy workloads for physicians and short consultations with patients [51]. Older patients often suffer from multiple and complex conditions. The inability of physicians to meet the demands of older patients for consultation time can translate into patient dissatisfaction [52, 53]. A mismatch between the needs of managing difficult physical and mental conditions of older patients and the training and professional skills obtained by primary care workers adds further complications to the situation [54, 55]. Physicians in primary care settings in China are often poorly equipped to confront the challenge [11].

Medical expenses were found to be negatively correlated with patient satisfaction, which is consistent with earlier studies $[48,56]$. In China, out-of-pocket payments still impose a great financial challenge to patients, despite the rapid expansion of health insurance coverage. A recent study showed that low reimbursement for tuberculosis (TB) treatments in China leads to heavy financial burdens on patients and their families. The new rural cooperative medical scheme has limited effects on alleviating the financial burdens of its enrollees and improving equity [57]. Adding to the complexity is the fact that insured patients tend to stay longer in hospital and receive more treatments than those who are uninsured [58]. Vincenzo [59] found that out-of-pocket expenses in China decreased only for high income individuals with good health status and monetary savings increased only for low income individuals with good health status.

\section{Gender composition of physicians is linked to patient satisfaction ratings}

We found that the gender composition of physicians in CHSs is associated with patient satisfaction in two ways. First, a higher percentage of male physicians weakens the negative association between patient age and patient satisfaction. Second, the gap in educational attainments between male and female physicians enhances the negative association of patient satisfaction with age.

A comparison of these findings with the results of other studies needs to be cautious. Previous studies found that female physicians tend to show more empathy through their non-verbal communications (i.e. smile and nodding), are more polite, and provide more advice to their patients than their male colleagues [20, 60-62]. However, this study did not link patient satisfaction with individual physicians. Gender composition was treated as an organizational feature.

The Chinese context may offer an explanation for our findings. Ting and colleagues [53] found that Chinese patients prefer an authoritarian approach rather than a more participatory approach in clinical decisions. They want health workers to act on their behalf without necessarily sharing the power in decision making. Both male and female patients prefer to choose a male doctor for consultations because they appear to have more "authority" than their female colleagues (a higher proportion of male physicians offers a better chance for such a choice) $[63,64]$. This belief is well aligned with the hierarchical Chinese culture where men play a dominant role in society. Professional work is usually considered to be the responsibility of men while women stay at home to look after the family. Although women were encouraged to enter the workforce when Chairman Mao seized power in 1949 and saw women as "holding up half of the sky", the stereotypical attitudes toward gender roles persist due to the thousands of years of influence of the Confucius philosophy. Interestingly, a study in California in the USA also demonstrated a higher degree of patient satisfaction with male doctors [65].

Male physicians are more likely to hold a university degree than female physicians in China. Understandably, 
patients tend to show more respect and satisfaction to those with a higher level of medical training. In general, $\mathrm{CHS}$ physicians in China are poorly trained. Many were transformed from "barefoot doctors" with limited vocational training [66]. Although university degree-based training for general practitioners started to attract attention in the 1980s [67], the current capacity of the university educational system is still very limited. For older patients with complex conditions, CHS physicians without a university degree can hardly meet their service needs [68].

\section{Conclusion}

A low level of patient satisfaction with CHSs is evident in the study setting. Women comprise more than half the physicians in CHSs in China. The negative link between patient satisfaction and the proportion of female physicians in CHSs is concerning. A significant gap in university qualifications between male and female physicians exists, which exacerbates the negative association between patient age and satisfaction ratings. Strengthening the professional training of female physicians may contribute to the improvement of patient satisfaction, especially for older patients.

It is important to note that patient satisfaction with CHSs is associated with both the individual characteristics of patients (such as age and medical expenses) and the collective characteristics of organizations (such as the gender composition of physicians). Comparisons of CHS organizations based on patient satisfaction data should consider risk adjustments for differences in physician and patient profiles. Furthermore, managers should be encouraged to put more effort into enhancing the professional training of female physicians, rather than developing policies that discriminate against female physicians $[69,70]$.

\section{Limitation}

This study was undertaken in 36 CHS organizations in Hubei province. The gender composition indicators are restricted to physicians in CHSs. The results should not be generalized to the entire hospital sector, nor to other healthcare workforce (such as nurses, pharmacists, allied health practitioners, and managers).

We have not been able to include patient diagnoses in the multi-level modelling due to unavailability of data. Further studies should consider patient casemix adjustment, if possible.

We established multi-level models using a summed patient satisfaction score based on three items because it is unreliable to transform a five-point Likert scale on a single item into a continuous measurement. We did not use the full scale of the PSQ-18 for concerns of overburdening the respondents. The $\beta$ coefficients are small although they are statistically significant.

\section{Abbreviations \\ CHS: Community health service; GDP: Gross domestic product; ICC: Intraclass correlation coefficients; MLE: Maximum likelihood estimation; \\ MSQ: Minnesota Satisfaction Questionnaire; PSQ-18: Patient satisfaction questionnaire short-form; TB: Tuberculosis}

\section{Acknowledgements}

We would like to thank the Health and Family Planning Commission of Hubei Province for their generous support.

\section{Funding}

The project was funded by a grant from the National Natural Science Foundation of China (NO: 71503075) and a grant from the scientific research program of Hubei Provincial Department of Education (NO: 15Y149,

D20142805). The funders have played no role in the design of the study and collection, analysis, and interpretation of data and in writing the manuscript.

\section{Availability of data and materials}

Please contact the corresponding author.

\section{Authors' contributions}

XC designed the study and drafted the manuscript. ZX participated in the design of the study, carried out the interviews, and helped in drafting the manuscript. CX participated in the data collection, data analyses, and writing of the manuscript. $\mathrm{CL}$ developed the analysis framework, led the interpretation of the results and revised the manuscript. All authors approved the final version of the manuscript.

\section{Ethics approval and consent to participate}

Ethics approval for this study was obtained from the Hubei University of Science and Technology Ethics Committee. The questionnaire surveys were anonymous. An informed consent form was distributed along with the questionnaire. Verbal consent was obtained from each participant before commencement of the face-to-face interview. The study protocol was approved by the ethics committee.

\section{Consent for publication}

Not applicable.

\section{Competing interests}

The authors declare that they have no competing interests.

\section{Publisher's Note}

Springer Nature remains neutral with regard to jurisdictional claims in published maps and institutional affiliations.

\section{Author details}

${ }^{1}$ Change Xiong, Department of Social Medicine and Health Management, School of Basic Medical Science, Hubei University of Science and Technology, 88 Xianning Road, Xianning 437100, People's Republic of China. ${ }^{2}$ Xiao Chen, Institute of Medicine, Hubei University of Science and Technology, 88 Xianning Road, Xianning 437100, People's Republic of China. ${ }^{3}$ Xinyuan Zhao, School of Clinic Medical Science, Hubei University of Science and Technology, Xianning 437100, People's Republic of China. ${ }^{4}$ Chaojie Liu, School of Psychology and Public Health, La Trobe University, Melbourne, VIC 3086, Australia.

Received: 16 April 2017 Accepted: 15 March 2018

Published online: 27 March 2018

\section{References}

1. Coulter A, Fitzpatrick R, Cornwell J. The point of care measures of patients' experience in hospital: purpose, methods and uses. King's Fund. 2009. Available at: https://www.researchgate.net/publication/230687403. Accessed 18 Nov 2017.

2. Fitzpatrick R. Satisfaction with healthcare. In: The experience of Illnessed Edn. London: Tavistock; 1984. p. 154-75. 
3. Kui-Son C, Hanjoon L, Chankon K, Sunhee L. The service quality dimensions and patient satisfaction relationships in South Korea: comparisons across gender, age and types of service. J Serv Mark. 2005;19(3):140-9.

4. Fang $\mathrm{P}$, Xiong $\mathrm{C}$. Existing problems on community health allocation in urban communities in China. Chinese Gen Pract. 2010;13(10A):3145-6.

5. Elliot R, Pearce CJ, Seifert C, Calder JD. Continuous infusion versus single bolus popliteal block following major ankle and hindfoot surgery: a prospective, randomized trial. Foot Ankle Int. 2010;31(12):1043-7.

6. Cleary P, McNeil B. Patient satisfaction as an indicator of quality care. Inquiry. 1988;25:25-36.

7. Minnick A, Roberts M, Young W, Kleinpell R, Marcantonio R. What influences patients reports of three aspects of hospital services? Med Care. 1997;35(4): 399-409.

8. Cohen G. Age and Health status in a patient satisfaction survey. Soc Sci Med. 1996:42(7):9.

9. Peck BM. Age-related differences in doctor-patient interaction and patient satisfaction. Curr Gerontol Geriatr Res. 2011:2011(8):1-10.

10. Leila M, Jean-Michel N, Christelle V, Bruno F, Pierre L, Isabelle G. Evidence of a non-linear influence of patient age on satisfaction with hospital care. Int J Qual Health Care. 2007;19(6):8.

11. Wallin E, Lundgren P-O, Ulander K, Von H, Christer S. Does age, gender or educational background effect patient satisfaction with short stay surgery? Ambul Surg. 2000;8(2):79-88.

12. Jensen JD, King AJ, Guntzviller LM, Davis LA. Patient-provider communication and low-income adults: age, race, literacy, and optimism predict communication satisfaction. Patient Educ Couns. 2010;79(1):30-5.

13. Fox JG, Storms DM. A different approach to sociodemographic predictors of satisfaction with health care. Soc Sci Med. 1981;15:557-64.

14. Karin DH, Sezgin C, Sophia MK. Patient satisfaction revisited: a multilevel approach. Soc Sci Med. 2009;69:68-75.

15. Hall J, Doman M. Patient sociodemographic characteristics as predictors of satisfaction with medical care: a meta-analysis. Soc Sci Med. 1990;30:811.

16. Hall A, Danielle BH, Roter DL. Patients' satisfaction with male versus female physicians: a meta-analisis. Med Care. 2011:49(7):611-7.

17. Bjorkman T, Hansson L, Svensson B, Berglund I. What is important in psychiatric outpatient care? Quality of care from the patient's perspective. Int J Qual Health Care. 1995;7:355-62.

18. Eagly A, Johnson B. Gender and leadership style: a meta-analysis. J Healthc Manag. 1980;112:102-24

19. Hall JA, Roter DL. Do patients talk differently to male and female physicians? Meta-analytic Rev Patient Educ Couns. 2002;48:217-24.

20. Harbinder S, Ann A, Laura S. The impact of gender dyads on doctor-patient communication: a systematic review. Patient Educ Couns. 2009;76:348-55.

21. Cypress BK. Characters of visits to female and male physicians. Vital Health Stat. 1980:13(49)

22. Mast MS, Hall JA, Roter DL. Disentangling physician sex and physician communication style: their effects on patient satisfaction in a virtual medical visit. Patient Educ Couns. 2007;68(1):16-22.

23. Anne-Cécile S, Cyrille D, Benoit L. Do gender differences affect the doctorpatient interaction during consultations in general practice? Results from the INTERMEDE study. Fam Pract. 2014;31(6):706-13.

24. Rubin H, Gandek B, Rogers W. Patients' ratings of outpatient visits in different practice settings: results from the medical outcomes study. JAMA. 1993;270:835.

25. Scholle S, Weisman C, Anderson R. Women's satisfaction with primary care: a new measurement effort from the PHS National Centers of excellence in Women's health. Womens Health Issues. 2000;10(1)

26. Meterko M, Mohr DC, Young GJ. Teamwork culture and patient satisfaction in hospitals. Med Care. 2004:492-8.

27. Liu C, Liu W, Wang Y, Zhang Z, Wang P. Patient safety culture in China: a case study in an outpatient setting in Beijing. BMJ Qual Saf. 2014;23(7):556-64.

28. Cliff B. Excellence in patient satisfaction within a patient-centered culture. Healthc Manag. 2012;57(5):157-9.

29. Tzeng H-M, Ketefian S, Redman RW. Relationship of nurses' assessment of organizational culture, job satisfaction, and patient satisfaction with nursing care. Int J Nurs Stud. 2002;39(3):79-84

30. Young GJ, Meterko M, Desai K. Patient satisfaction with hospital care: effects of demographic and institutional characteristics. Med Care. 2000; 38(3):325-34.

31. Fleming G. Hospital structure and consumer satisfaction. Health Serv Res. 1981;16:43-61.
32. Regula NC, Judith A, Johannes B. Gender differences in physicians' communicative skills and their influence on patient satisfaction in gynaecological outpatient consultations. Soc Sci Med. 2008;66:1474-83.

33. Alexander B, Patricia H, Patrick AB. Doctor-patient gender concordance and patient satisfaction in interpreter-mediated consultation: an exploratory study. J Travel Med. 2008:15(1):1-5.

34. National Health and Family Planning Commission. Chinese Health Statistics Yearbook 2013. http://www.nhfpc.gov.cn/htmlfiles/zwgkzt/ptjnj/year2013/ index2013.html. Accessed 6 Apr 2015.

35. Australian Institute of Health and Welfare. What types of medical practitioners are there? https://www.aihw.gov.au/reports/workforce/medicalpractitioners-workforce-2015/contents/what-types-of-medical-practitionersare-there. Accessed 24 Aug 2016.

36. Service Canada. Human resources for health. https://www.cihi.ca/sites/ default/files/document/physicians in canada_phys2016_en.pdf. Accessed 16 Nov 2017.

37. NHS. General and Personal Medical Services, England. http://www.hscic.gov. uk/catalogue/PUB16934/nhs-staf-2004-2014-gene-prac-rep.pdf. Accessed 16 Mar 2016.

38. American Board of Family Medicine. Gender statistics. https://www.theabfm. org/about/stats gender.aspx. Accessed 16 Mar 2016.

39. Hubei Health and Family Planning Commission. Health development bulletin of Hubei Province. http://www.hbwsjs.gov.cn/detail/ 20150424111112699001.html. Accessed 20 Oct 2016.

40. Ali BM, Reisel WD. Relating patient satisfaction to nurses' job satisfaction, job security, and obedience OCBs. Int J Pharm Healthc Mark. 2014; 8(1):47-61.

41. Jill M. The feelgood factor: Jill Maben examines the evidence for a link between staff wellbeing and the quality of patient care. Nurs Stand. 2010; 24(30):70.

42. Weiss DJ, Dawis RV, England GW, Lofquist LH. Manual for the Minnesota satisfaction questionnaire.Vol 22, Minnesota Studies in Vocational Rehabilitation. Minneapolis: University of Minnesota, Industrial Relations Center; 1967.

43. Fields DL. Taking the measure of work: a guide to validated scales for organizational research and diagnosis. Thousand Oak: Sage; 2002

44. Marshall GN, Hays RD. The patient satisfaction questionnaire short-form show (PSQ-18): Santa Monica, Rand, CA; 1994

45. Sixma HJ, Campen CV, Peters KL. Quality of care from the perspective of elderly people: the QUOTE-elderly instrument. Age Ageing. 2000;29:173-8.

46. Fletcher RH, O'Malley M, Earp JA, Littleton TA, Fletcher SW, Greganti MA, Davison RA, Taylor J. Patients' priorities for medical care. Med Care. 1983; 21(2):234-42.

47. Ware JEJ, Davies-Avery A, Stewart AL. The measurement and meaning of patient satisfaction: a review of the recent literature. Health Med Care Serv Rev. 1978;1(1):1-15.

48. Hall J, Dornan M. What patients like about their medical care and how often they are asked: a meta-analysis of the satisfaction literature. Soc Sci Med. 1988;27(9):935.

49. Lai GQ, Wang YP, Chen J, Zhang W, Chen HL. Effect of implementation of initial medical care at community in Shenzhen and thinking. Chinese Gen Pract. 2009;12(2A):202-5.

50. Fang Z, Yao Y. Study on the outpatient's satisfaction of the community health service in Wuhan. Med Soc. 2009;22(12):27-8.

51. Zhang $Y$, Zhang $D, B o H$. Discussion on training of general practitioners in China present situation. Contin Med Educ. 2015;29(9):3-5.

52. Carr-Bains S, Nightingale A, Ballard K. Patients' experiences and satisfaction with out-of-hours GP home visiting provided by a GP cooperative. Fam Pract. 2011;28(1):88-92

53. Ting $X$, Yong $B$, Yin $L$, Mi T. Patient perception and the barriers to practicing patient-centered communication: a survey and in-depth interview of Chinese patients and physicians. Patient Educ Couns. 2016;99(3):364-9.

54. Sun J. Mental problems of empty nesters in rural areas in China and its community nursing countermeasures. Chinese Gen Pract Nurs. 2014; 12(22):2030-2.

55. Wang $Y$, Sha $S$, Jiang $C$. Study on influence of individual intervention on psychological symptoms and quality of life of empty nesters in community of shanghai city. Chin Nurs Res. 2014;28(3B):970-2.

56. Huerta TR, Hartle CA, Ford EW. Measuring patient satisfaction's relationship to hospital cost efficiency: can administrators make a difference? Health Care Manag Rev. 2016;41(1):56-63. 
57. Li X, Yao P, Shuang Y. The impact of new cooperative medial scheme on financial burden of tuberculosis patients:evidence from six countries in China. Infect Dis Poverty. 2016;5:8-19.

58. Liu K, Wu Q, Liu J. Examining the association between social health insurance participation and patients' out-of-pocket payments in China: the role of institutional arrangement. Soc Sci Med. 2014;113:95-103.

59. Vincenzo A, Agar B, Noemi P. The health care system reform in China: effects on out-of-pocket expenses and saving. China Econ Rev. 2015;34(7): 182-95.

60. Flocke SAGV. Physician and patient gender concordance and the delivery of comprehensive clinical preventive services. Med Care. 2005:43:486-92.

61. Hall J, Irish J, Roter D, Ehrlich C, Miller L. Gender in medical encounters: an analysis of physician and patient communication in a primary care setting. Health Psychol. 1994;13:384-92.

62. Roter D, Hall J, Aoki Y. Physician gender effects in medical communication: a meta-analytic review. JAMA. 2002;288:756-64.

63. Zaghloul AA, Youssef AA, El-Einein NY. Patient preference for providers'gender at a primary health care setting in Alexandria, Egypt. Saudi Med J. 2005;26(1):90-5.

64. Hall JA, Gulbrandsen P, Dahl FA. Physician gender, physician patientcentered behavior, and patient satisfaction: a study in three practice setting within a hospital. Patient Educ Couns. 2014:95:313-8.

65. Lund JD, Rohrer JE, Goldfarb S. Patient gender preferences in a large military teaching hospital. Obstet Gynecol. 2005:4:747-50.

66. Szecsenyi J, Goetz K, Campbell S, Broge B, Reuschenbach B, Wensing M. Is the job satisfaction of primary care team members associated with patient satisfaction? BMJ Qual Saf. 2011;20(6):508-14.

67. Ma X, Dai T, Yang SX. International experiences and implications of the practice and service mode of general practitioners. Chinese J Health Policy. 2015;8(2):13-8

68. Su L, Zhang Q, Li W. A survey of knowledge and skills on healthy education by junior general practitioners and specialists. China High Med Educ. 2014; 12:83-4.

69. Celik H, Lagro-Janssen T, Widdershoven G, Abma T. Bringing gender sensitivity into healthcare practice: a systematic review. Patient Educ Couns. 2011;84:143-9

70. Sen G, Stlin P. Women and gender equity knowledge network. Unequal, unfair, ineffective and inefficient gender inequity in health: why it exists and how we can change it. Final report to the WHO Commission on social determinants of health. Geneva: World Health Organization; 2007.

\section{Submit your next manuscript to BioMed Central and we will help you at every step:}

- We accept pre-submission inquiries

- Our selector tool helps you to find the most relevant journal

- We provide round the clock customer support

- Convenient online submission

- Thorough peer review

- Inclusion in PubMed and all major indexing services

- Maximum visibility for your research

Submit your manuscript at www.biomedcentral.com/submit 